\title{
Can prolonged sick leave after gynecologic surgery be predicted? An observational study in the Netherlands
}

\author{
Hans A. M. Brölmann • Antonie Vonk Noordegraaf • \\ David J. Bruinvels · Riekie H. C. de Vet • \\ Amarantha A. Dirksz · Judith A. F. Huirne
}

Received: 11 June 2008/ Accepted: 26 November 2008/Published online: 1 January 2009

(C) The Author(s) 2008. This article is published with open access at Springerlink.com

\begin{abstract}
Background Sick leave frequently has been used as an outcome to evaluate minimal invasive surgery compared with conventional open surgery. However, sick leave is determined not only by the surgical approach. Recently, a postoperative recovery-specific quality-of-life questionnaire, the Recovery Index (RI-10), has been developed and validated. This study investigated the relation of the Recovery Index 10, the RI-6 (a subset of 6 questions), and the type of surgery to sick leave.

Methods The study enrolled 46 patients with a paid job scheduled for elective gynecologic surgery, who filled out the RI-10. After 8 weeks, the patients were approached by telephone to give information on their return to work.

Results Of the 46 patients, $23(50 \%)$ returned to work completely after 8 weeks, 14 (30\%) resumed work partly, and $9(20 \%)$ did not resume work at all. In the analysis, the patients who completely returned to work were compared with those who did not return or partially returned. Recovery as expressed in the RI-6 improved with time after surgery. It appeared that the measurement 2 weeks after surgery showed the best discriminative capacity to predict sick leave after 8 weeks, with an area under the curve of 0.88 (confidence interval, 0.74-1.03). The subjective postoperative recovery as expressed by the RI-6 is more
\end{abstract}

H. A. M. Brölmann $(\bowtie) \cdot$ A. Vonk Noordegraaf .

J. A. F. Huirne

Department of Obstetrics and Gynecology, VU University

Medical Center, De Boelelaan 1117, 1181HV Amsterdam,

The Netherlands

e-mail: h.brolmann@vumc.nl

D. J. Bruinvels - R. H. C. de Vet - A. A. Dirksz Department of EMGO, VU University Medical Center, De Boelelaan 1117, 1181HV Amsterdam, The Netherlands closely related to the type of surgery $(p=0.001)$ sick leave is $(p=0.14)$.

Conclusions The subjective recovery scored by the patient on a questionnaire of six questions is a better outcome than sick leave for evaluating surgical approaches. If administered 2 weeks after surgery, it may predict prolonged sick leave.

Keywords Gynecology · Laparoscopy ·

Postoperative recovery - Quality of life - Sick leave

The main objective of laparoscopic surgery is to reduce postoperative recovery time by making smaller wounds than laparotomy. Consequently, patients can resume their daily activities and work sooner. In this way, the laparoscopic approach may reduce the sick leave of workers and their loss of productivity after surgery. Sick leave has been used frequently as an outcome for comparing minimally invasive surgery with conventional open surgery. Although the direct costs resulting from disposable instruments and the operating time may be greater with the laparoscopic approach, the profit in terms of productivity costs may be substantial, possibly compensating for the higher direct hospital costs $[1,2]$.

However, sick leave is determined not only by the surgical approach, but also by subjective recovery in combination with local and personal factors such as employment, job satisfaction, psychological well-being, and given recommendations (expectations) about work resumption, regardless of the surgical technique. Convalescence recommendations given by medical professionals show substantial variability and are not evidence based [3]. Consequently, the advantage of the laparoscopic approach might not be fully realized. 
Recently, a postoperative recovery-specific quality-oflife questionnaire, the Recovery Index (RI-10), has been developed and validated in terms of internal consistency and construct validity [4]. The Recovery Index mainly measures the subjective recovery, and we therefore hypothesize that it will be a more appropriate outcome measure than sick leave for studying the effects of the surgical approach (e.g., laparoscopic vs open techniques).

Because the subjective recovery is or should be fundamental for return to work, we expect that a low score on the Recovery Index in the early postoperative phase predicts a prolonged sick leave. Comparable, although targeting quality of life instead of sick leave, is the study of Myles et al. [5], who demonstrated with cardiac surgery patients that a poor recovery, expressed as a recovery-specific quality-of-life score, in the hospital predicted a poor generic quality of life at 3 months.

The first objective of this study was to investigate what RI-10 questions and what time of administration have value for predicting prolonged sick leave among workers who undergo gynecologic surgery for benign reasons in the Amsterdam region of the Netherlands. The second objective was to validate this possible subset of questions in terms of internal consistency and construct validity by relating it to postoperative time and type of surgery.

\section{Materials and methods}

After approval of the institutional review board and written informed consent, 52 patients scheduled for elective gynecologic surgery in 2006 at the VU University Medical Center in Amsterdam, The Netherlands, who had a paid job, were entered into the study. Patients with oncologic disease, acute disease that required surgery, or ambulatory surgery were excluded.

Before surgery, the patients filled out questionnaires that provided data on their employment and education level. The Recovery Index-6 (RI-6) is a subset of questions from the Recovery Index-10 (RI-10). The RI-10 is a quality-oflife questionnaire for measuring subjective postoperative recovery. Patients can indicate their level of agreement with each item on a scale from 1 (completely agree) to 5 (completely disagree). The total score of the RI-10, controlled for positive and negative formulations, is divided by 10 and ranges from 1 to 5 . A score of 1 means complete subjective recovery. The RI-10 has been validated recently [4].

The RI-10 was self-administered by the patients $1,2,3$, 4 , and 6 weeks after surgery. The selection of six questions from the RI 10 was based on their individual association with sick leave. Additionally, after 8 weeks, the patients were approached by telephone to give information on their
Table 1 Classification of gynecologic operations according to the gravity of surgery

\begin{tabular}{ll}
\hline - Minor surgery & Number \\
○ Diagnostic laparoscopy & 3 \\
○ Laparoscopic adnexal surgery & 3 \\
○ Laparoscopic adhesiolysis & 3 \\
- Intermediate surgery & \\
○ Total laparoscopic hysterectomy & 5 \\
O Laparotomy for adnexal surgery & 9 \\
○ Vaginal hysterectomy & 9 \\
- Major surgery & \\
- Total abdominal hysterectomy & 9 \\
○ Excision endometriosis stage 3-4 & 5 \\
\hline
\end{tabular}

return to work. Their surgery was classified as minor, intermediate, and major, as stated in Table 1.

The data were analyzed using SPSS 15.0 (SPSS inc., Chicago, IL, USA). Cronbach's alpha was calculated as a measure of the internal consistency of the RI-6 based on the average inter-item correlation. The chi-square statistic was used to assess the relation of the RI-6 and sick leave to the type of surgery. Receiver operator characteristics (ROC) curves were constructed to display the value of the RI-6 measured at different intervals after surgery for predicting return to work after 8 weeks.

The ROC curves were constructed using the scores of the RI- 6 at 1, 2, 3, 4, and 6 weeks. By calculating the area under the curve (AUC), the most accurate ROC curve was identified. Subsequently, the most accurate cutoff score of the curve was established, and test characteristics (sensitivity and specificity) were calculated. Univariate analyses were performed to assess the relation between various variables and sick leave after 8 weeks. Variables showing a $p$ value less than 0.2 were included in the multivariate logistic regression analysis. Statistical tests were performed using a two-sided approach, and a $p$ value less than 0.05 was considered significant.

\section{Results}

Because not all 52 patients completed all the questionnaires, 46 patients were available for analysis. Of the 46 patients, 23 (50\%) returned to work completely after 8 weeks, 14 (30\%) resumed their work partly, and $9(20 \%)$ did not resume work at all. In the analysis, the patients with complete return to work were compared with those who did not return or returned partly.

A subset of six questions was used because in the logistic regression analysis, only these questions, if controlled for type of surgery, had an independent value for predicting return to work after 8 weeks (Table 2). 
Table 2 Capacity of the distinguished questions in the Recovery Index 10 (RI-10), controlled for type of surgery, to predict sick leave for 46 patients after gynecologic surgery ${ }^{\mathrm{a}}$

\begin{tabular}{|c|c|c|}
\hline Questions RI-10 & Odds ratio $^{\mathrm{b}}$ & $p$ Value \\
\hline Slight exertion makes me feel tired & 2.6 & 0.006 \\
\hline During the day I need to rest regularly & 3.0 & 0.001 \\
\hline Even without activity, I am bothered by abdominal pain & 1.8 & 0.025 \\
\hline Any light work (e.g., making coffee) exhausts me & 2.2 & 0.008 \\
\hline I feel completely recovered after surgery & 0.14 & 0.001 \\
\hline I can finish my daily activities at home without effort & 0.2 & 0.003 \\
\hline Since the operation, I have problems sleeping & 1.1 & 0.5 \\
\hline Surgery and recovery have not been as uneventful as I expected & 1.3 & 0.2 \\
\hline I had a lot of pain after surgery & 1.4 & 0.13 \\
\hline The complaints for which I had surgery are completely resolved & 0.8 & 0.5 \\
\hline
\end{tabular}

${ }^{a}$ Patients indicated their agreement on a Likert scale from 1 (completely agree) to 5 (completely disagree). The total score was divided by 10 and ranges from 1 to 5

${ }^{\mathrm{b}}$ Odds ratio for return to work, controlled for type of surgery by logistic regression analysis

Recovery as expressed in the RI-6 after $1,2,3,4$, and 6 weeks improved with time after surgery and differed between patients who completely returned to work after 8 weeks and those who did not (Fig. 1). Figure 2 shows the predictive value of the RI-6, expressed as an ROC curve assessed at various moments after surgery. It appeared that the measurement 2 weeks after surgery showed the best discriminative capacity to predict sick leave after 8 weeks, with an AUC of 0.88 (confidence interval [CI], 0.74-1.03). The most predictive RI-6 score (at week 2) then was chosen as the subjective recovery instrument. The Cronbach alpha of the RI-6 was 0.94 , and the inter-item correlation ranged from 0.71 to 0.92 .

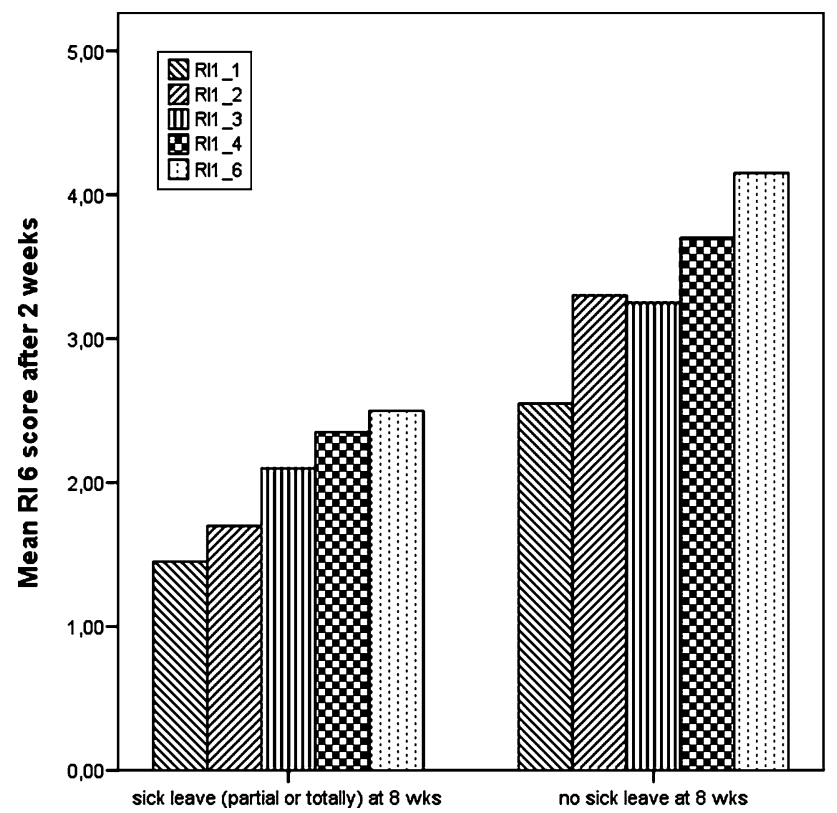

Fig. 1 The mean RI-6 score of 46 patients after gynecologic surgery according to postoperative weeks $(1,2,3,4$, and 6$)$ and return to work after 8 weeks
Table 3 shows that the subjective postoperative recovery, as expressed by the RI-6, is more closely related to the type of surgery $(p=0.001)$ than sick leave is $(p=0.14)$. With regard to prediction of return to work, we found no significant association of return to work with level of education or type of work (sedentary, light, heavy), as shown in Table 4. The way of employment (self-employment vs employee) and the type of surgery showed $p$ values less than 0.2 . We did not include the way of employment in the multivariate analyses because of the small number of self-employed patients.

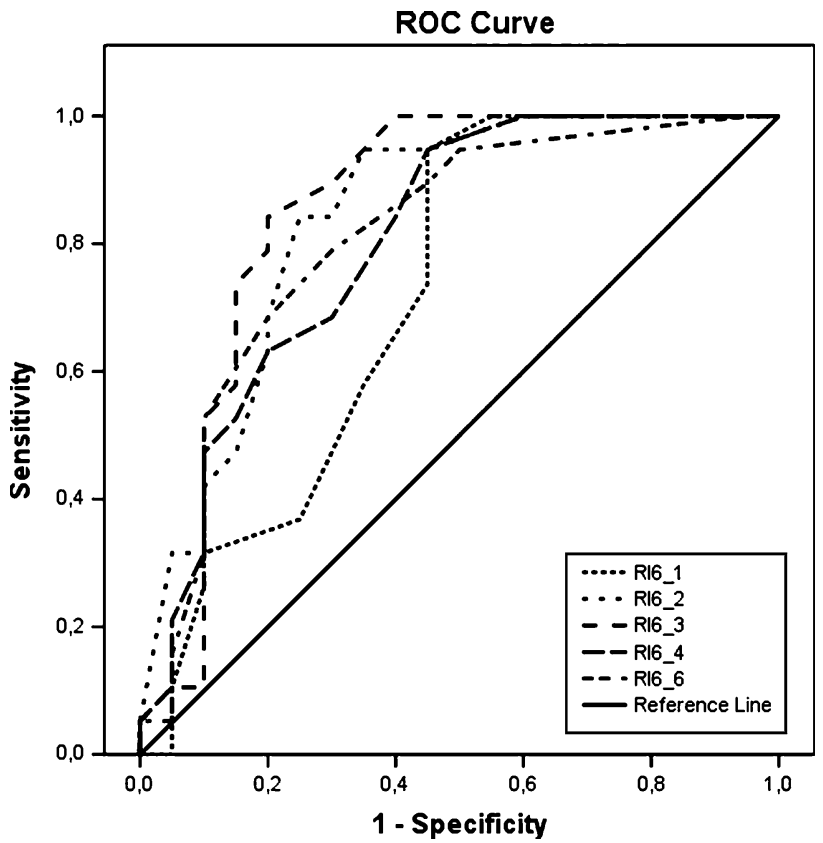

Fig. 2 Receiver operating characteristic curve of the Recovery Index 6 (RI-6) scores 1, 2, 3, 4, and 6 weeks after surgery predicting complete return to work after 8 weeks for 46 patients after gynecologic surgery 
Table 3 Cross-tabulation of the extent to which the type of surgery is associated with the Recovery Index 6 (RI-6) and with the sick leave data for 46 patients scheduled for elective gynecologic surgery

${ }^{a}$ No return or a partial return to work

\begin{tabular}{lllll}
\hline & Minor surgery $(n)$ & Intermediate surgery $(n)$ & Major surgery $(n)$ & $p\left(\chi^{2)}\right.$ \\
\hline RI-6 & & & & \\
$-<1$ & 2 & 4 & 5 & 0.001 \\
$-1-2$ & 6 & 12 & 2 & \\
$-2-3$ & 1 & 7 & 2 & \\
$->3$ & 5 & 0 & 0 & 0.14 \\
Sick leave & & & & \\
- Yes $^{\text {a }}$ & 4 & 13 & 6 & \\
- No & 10 & 10 & 3 & \\
\hline
\end{tabular}

Table 4 Sick leave or a partial return to work according to preoperative variables for 46 patients undergoing benign gynecologic surgery

\begin{tabular}{|c|c|c|c|}
\hline Variable & Sick leave $^{\mathrm{a}}(n)$ & Return to work $(n)$ & $p$ Value \\
\hline \multicolumn{4}{|l|}{ Employment ${ }^{\mathrm{b}}$} \\
\hline - Self-employed & 0 & 5 & \multirow[t]{2}{*}{0.05} \\
\hline - Employed & 22 & 18 & \\
\hline \multicolumn{4}{|c|}{ Level of education ${ }^{\mathrm{b}}$} \\
\hline - Low & 5 & 6 & \multirow[t]{3}{*}{0.96} \\
\hline - Middle & 6 & 6 & \\
\hline - High & 11 & 11 & \\
\hline \multicolumn{4}{|l|}{ Type of work ${ }^{b}$} \\
\hline - Light & 11 & 13 & \multirow[t]{3}{*}{0.47} \\
\hline - Moderate & 5 & 7 & \\
\hline - Heavy & 6 & 3 & \\
\hline \multicolumn{4}{|l|}{ Type of surgery } \\
\hline - Minor & 4 & 10 & \multirow[t]{3}{*}{0.14} \\
\hline - Intermediate & 13 & 10 & \\
\hline - Major & 6 & 3 & \\
\hline \multicolumn{4}{|l|}{ Recovery Index 6} \\
\hline$\bullet<1$ & 9 & 2 & \multirow[t]{4}{*}{0.003} \\
\hline - $1-2$ & 12 & 8 & \\
\hline - $2-3$ & 2 & 8 & \\
\hline$\cdot>3$ & 0 & 5 & \\
\hline
\end{tabular}

${ }^{a}$ No return or a partial return to work

${ }^{\mathrm{b}}$ One patient did not answer this question

Using the ROC curve, we found an RI-6 score of 1.7 to be the optimal cutoff point, with its sensitivity of $68 \%$ and its specificity $74 \%$ regarding return to work after 8 weeks. A low score was associated with $25 \%$ of the patients completely returned to work (predictive value, 75\%) compared with $72 \%$ of the patients in relation to a high score.

Logistic regression was performed using the type of surgery and the RI-6 score after 2 weeks as independent variables. The RI-6 was dichotomized due to the small number of subjects, with 1.7 used as a cutoff level. Only the RI- 6 was an independent predictor of return to work, although the number of subjects was small (Table 5).
Table 5 Logistic regression using return to work after 8 weeks as the dependent variable and using type of surgery and RI-6 score as independent variables for 46 patients after gynecologic surgery ${ }^{\mathrm{a}}$

\begin{tabular}{|c|c|c|c|}
\hline Predicting factor & Odds rate $^{\mathrm{b}}$ & $\mathrm{CI}$ & $p$ Value \\
\hline Type of surgery ${ }^{c}$ & 3.4 & $0.4-32$ & 0.28 \\
\hline RI-6 index & 6.5 & $1.9-21$ & 0.002 \\
\hline
\end{tabular}

CI confidence interval

${ }^{a}$ Variable or variables entered in step 1: type of surgery and RI-6 index

b The odds rate represents the ratio change in the odds of not being completely returned to work for a one-unit change in the predicting factor

c Minor, intermediate, and major surgery

\section{Discussion}

Sick leave 8 weeks after gynecologic surgery is unexpectedly high (median. 50\%) in a working population of Dutch patients with benign disease. No return to work 8 weeks after elective gynecologic surgery can be considered generally as a prolonged sick leave. The RI-6 shows the score correlates well with the time until return to work after surgery. Because the subjective recovery (RI-6) is more closely related than sick leave to the type of surgery (Table 3), it seems to be a better tool for comparing laparoscopic and open surgery, confirming that sick leave may not be based on recovery alone.

Reasons for the high proportion of patients with a prolonged sick leave after surgery cannot be given because they were not addressed in the questionnaire. However, many women reported tiredness and a need for rest during the day (questions 1 and 2 of the RI-10). The lack of consistent recommendations by professionals about return to work may not promote early recovery.

There is a substantial correlation between the RI- 6 after 2 weeks and sick leave at 8 weeks. In the multivariate analysis, the RI-6 at postoperative week 2 was able to predict prolonged sick leave with considerable accuracy. Patients with an RI-6 score below 1.7, reflecting an obstructed recovery, have a $25 \%$ probability of being at good internal consistency and construct validity because 
work after 8 weeks, and patients with an RI-6 score higher than 1.7 have a $72 \%$ probability of being completely at work after 8 weeks. However, the number of subjects was too small for a logistic regression with all potentially predictive variables. For example, in the univariate analysis, patients who were self-employed (employers) apparently were at less risk for prolonged sick leave.

This study had some methodologic shortcomings. Questions from the original RI-10 [4] were selected for the RI-6 based on their value for predicting return to work. Therefore, the findings need to be externally validated by their confirmation in another population. This study currently is underway. The sick leave was reported by the patients and not retrieved from the employer. This may affect the reliability of the data. Finally, presentation of the return-to-work data is more appropriate by life table analysis, but we had no data on the number of sick leave days.

Several reasons exist for prolonged sick leave after surgery. One reason is varying information on convalescence by medical professionals. In a survey to collect current opinions among surgeons and general practitioners about the time a patient should be absent from work, a wide range of times off work were recommended [6]. Also in randomized trials on laparoscopic hysterectomy, the return to work varied from 10 [7] to 39 days [8], at least partly reflecting varying recommendations with regard to sick leave. It has been shown that standardizing postoperative recommendations can result in shortening of the sick leave [9, 10].

In studies comparing minimally invasive and conventional surgery, the complication rate, admission time, and return to daily activities and work usually are given as outcomes, with the assumption that both approaches are equally effective. Rarely is the quality of life reflecting the subjective recovery used. In a systematic review of randomized trials comparing laparoscopic hysterectomy with total abdominal hysterectomy, only in 7 of 30 trials was quality of life used as an outcome, and only in 1 trial was it used as the primary outcome [11]. In four studies, validated questionnaires were used, with a difference between the two study arms found in only two studies. This may be explained by the fact that generic quality-of-life questionnaires such as the Short Form 36 or the EuroQol 5 were used.

It is well known that generic questionnaires are not very sensitive in specific situations. Therefore, we recommend the use of a validated postoperative recovery-specific questionnaire that is optimally able to measure recovery after different surgical approaches.

We have shown that the RI-6 is able to predict sick leave after surgery. This enables the detection of patients with slow recovery and prolonged return to work already at 2 weeks after surgery.

In conclusion, we found the subjective recovery scored by the patient in a questionnaire of six questions to be a better outcome measure than sick leave for evaluating a surgical approach (e.g., laparoscopy vs laparotomy). If administered 2 weeks after surgery, it may predict prolonged sick leave. In this way, patients at risk for prolonged sick leave can be subjected to an ergonomic intervention, thus doing justice to the advantages of the laparoscopic surgery. Larger-scale studies are needed to confirm the predictive value and validity of the RI-6.

Open Access This article is distributed under the terms of the Creative Commons Attribution Noncommercial License which permits any noncommercial use, distribution, and reproduction in any medium, provided the original author(s) and source are credited.

\section{References}

1. Ellstrom M, Ferraz-Nunes J, Hahlin M, Olsson JH (1998) A randomized trial with a cost-consequence analysis after laparoscopic and abdominal hysterectomy. Obstet Gynecol 91:30-34

2. Olsson JH, Ellstrom M, Hahlin M (1996) A randomised prospective trial comparing laparoscopic and abdominal hysterectomy. Br J Obstet Gynaecol 103:345-350

3. Ottesen M, Moller C, Kehlet H, Ottesen B (2001) Substantial variability in postoperative treatment and convalescence recommendations following vaginal repair: a nationwide questionnaire study. Acta Obstet Gynecol Scand 80:1062-1068

4. Kluivers KB, Hendriks JC, Mol BW, Bongers MY, Vierhout ME, Brolmann HA et al (2008) Clinimetric properties of 3 instruments measuring postoperative recovery in a gynecologic surgical population. Surgery $144: 12-21$

5. Myles PS, Hunt JO, Fletcher H, Solly R, Woodward D, Kelly S (2001) Relation between quality of recovery in hospital and quality of life at 3 months after cardiac surgery. Anesthesiology 95:862-867

6. Majeed AW, Brown S, Williams N, Hannay DR, Johnson AG (1995) Variations in medical attitudes to postoperative recovery period. BMJ 311:296

7. Deprest JA, Munro MG, Koninckx PR (1995) Review on laparoscopic hysterectomy. Zentralbl Gynakol 117:641-651

8. Richardson RE, Bournas N, Magos AL (1995) Is laparoscopic hysterectomy a waste of time? Lancet 345:36-41

9. Bisgaard T, Klarskov B, Rosenberg J, Kehlet H (2001) Factors determining convalescence after uncomplicated laparoscopic cholecystectomy. Arch Surg 136:917-921

10. Ottesen M, Sorensen M, Kehlet H, Ottesen B (2003) Short convalescence after vaginal prolapse surgery. Acta Obstet Gynecol Scand 82:359-366

11. Kluivers KB, Johnson NP, Chien P, Vierhout ME, Bongers M, Mol BW (2008) Comparison of laparoscopic and abdominal hysterectomy in terms of quality of life: a systematic review. Eur J Obstet Gynecol Reprod Biol 136:3-8 